\title{
TIME-DEPENDENT, LATTICE APPROACH TO ATOMIC COLLISIONS
}

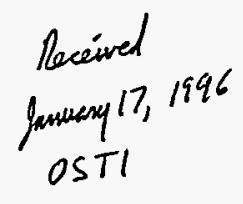

\author{
David R. Schultz

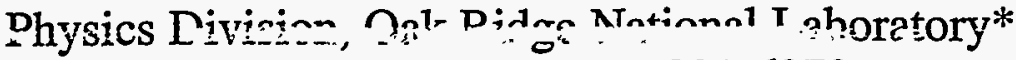 \\ Oak Ridge, Tennessee 37831-6373
}

\author{
to be published in Proceedings of \\ XIX International Conference on the Physics of Electronic \\ and Atomic Collisions \\ Whistler, Canada \\ July 26 - August 1, 1995
}

\begin{abstract}
*Managed by Lockheed Martin Energy Systems under Contract DE-AC05-84OR21400 with the U.S. Department of Energy.
\end{abstract}

\section{DISCLAIMER}

This report was prepared as an account of work sponsored by an agency of the United States Government. Neither the United States Government nor any agency thereof, nor any of their employees, makes any warranty, express or implied, or assumes any legal liability or responsibility for the accuracy, completeness, or usefulness of any information, apparatus, product, or process disclosed, or represents that its use would not infringe privately owned rights. Reference herein to any specific commercial product, process, or service by trade name, trademark, manufacturer, or otherwise does not necessarily constitute or imply its endorsement, recommendation, or favoring by the United States Government or any agency thereof. The views and opinions of authors expressed herein do not necessarily state or reflect those of the United States Government or any agency thereof.

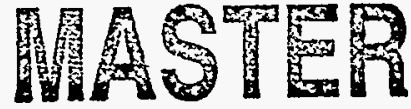

"The submitted manuscript has been authored by a contractor of the U.S. Government under Contract No. DE-AC05840R21400. Accordingly, the U.S. Government retains a nonexclusive, royaltyfree license to publish or reproduce the published form of this contribution, or allow others to do so, for U.S. Government purposes." 


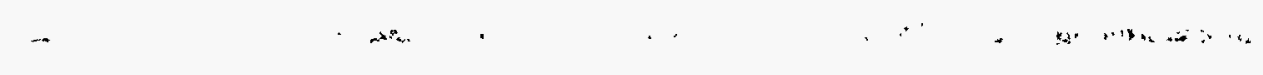

$1+\cdots \cdot i^{\prime} \cdot$ 


\title{
Time-Dependent, Lattice Approach to Atomic Collisions
}

\author{
David R. Schultz \\ Physics Division, Oak Ridge National Laboratory \\ Oak Ridge, Tennessee 37831-6373
}

\begin{abstract}
Recent progress in developing and applying methods of direct numerical solution of atomic collision problems is described. Various forms of the three-body problem are used to illustrate these techniques. Specifically, the process of ionization in proton-, antiproton-, .. I , 2, 2, :ranging in computational intensity from collisions simulated in two spatial dimensions to treatment of the three-dimensional, fully correlated two-electron Schrödinger equation. These examples demonstrate the utility and feasibility of treating strongly interacting atomic systems through time-dependent, lattice approaches.
\end{abstract}

\section{INTRODUCTION}

It would not be unusual for a paper describing methods of treating atomic collisions on a numerical lattice to begin with a sentence such as "With the advent of contemporary supercomputing resources, direct solution of the timedependent Schrödinger equation for collisions of ions or electrons with atoms has finally become a feasible undertaking." Indeed, a number of works have appeared in which pilot or proof-of-principle calculations have been carried out demonstrating that multidimensional wavefunctions and operators may be discretized and the collision dynamics faithfully described (see e.g. Figure 1). But it has also been a goal of atomic physicists to bring these techniques to bear on the very accurate treatment of fundamental atomic collision problems on a more routine basis.

What do we mean by routine and why in particular should we seek the application of calculations performed on a numerical grid?

The goal of making lattice approaches part of the atomic physicist's tool kit alongside perturbation theory, more traditional close coupling schemes, and classical scattering theory, requires that individual calculations be accomplished in times, say, shorter than a day. Only then could results which must be repeated for many impact parameters or angular momenta, or to prove convergence with lattice size or grid spacing be said to be routine. The objective is thus techniques which can significantly impact physical interpretation of phenomena, and to do that often requires computing probabilities or cross sections over a range of collision energies or geometries. It is this

(C) 1993 American Institute of Physics 


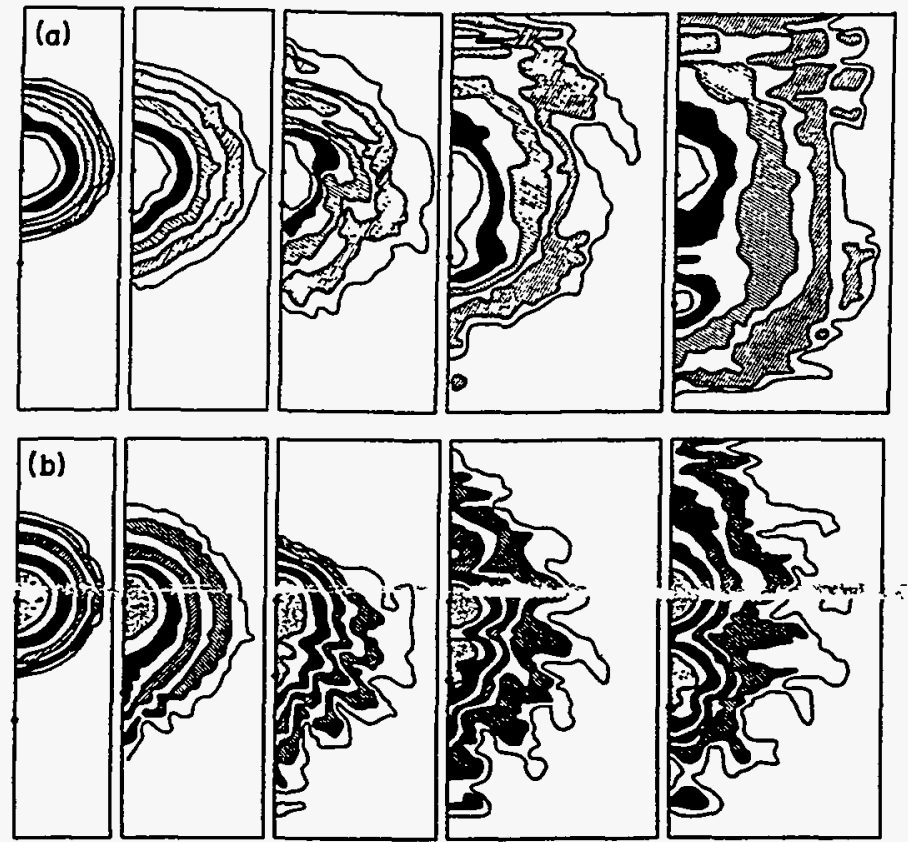

FIG. 1. Contour density plots illustrating the time evolution of the electronic wavefunction for (a) $\mathrm{H}^{+}$and (b) $\mathrm{C}^{6+}$ with atomic hydrogen for several time slices throughout the collision. These calculations (1)were performed in 1982 using the fastest available supercomputers, but still covered only a very small collision volume and relied on the approximation of axial decoupling to reduce the problem to two spatial dimensions.

desire for physical insight that also coincides with one of the advantages of the method. That is, the lattice approach lends itself to a very ready visualization of the collision process in time. Furthermore, many lattice methods rely on flexible basis functions so that a very wide variety of phenomena can be represented. They also seek to solve as directly as possible the equations governing the interaction (e.g. the Schrödinger or Hartree-Fock equations) for even strongly interacting systems, and therefore provide a powerful nonperturbative treatment for inherently time-dependent problems.

Here we report on our recent progress in makin routine and in deriving insight from them regarding fundamental atomic collisions. In particular, we describe our treatment of three, three-body problems, namely ionization in proton-, antiproton-, and electron-impact of atomic hydrogen. 


\section{MODEL $\mathrm{H}^{+}+\mathrm{H}$ COLLISIONS}

Recently, there has been great activity in developing theories of ionization in ion-atom collisions especially in the low- to intermediate-energy regime, motivated in large part by new experimental measurements. The most elementary quantum mechanical theory of ionization, the first Born approximation, has been perhaps remarkably successful but simply treats the electron as being ejected into the continuum of the residual target ion through a perturbative interaction with the projectile. Evidence that this theory is inadequate to describe the full ejected electron spectrum has been found for some time.

A number of works (see e.g. (2-6)) in the past decade have therefore emphasized the need to treat the electron ejected in ionization as moving in the combined field of both the target and projectile ions. Such theories include the classical trajectory Monte Carlo (CTMC) method (6.7), the continuumdistorted-wave-eikonal-initial-state (CDWEIS) approximation $(2,5)$ and the strong-potential-Born (DSPB) approximation (8). These theories treat the electron as being ejected under the combined influence of both the target and projectile ions. To varying degrees each has been successful in describing the ejected electron spectrum for a rather wide range of collision energies. For example, at intermediate collision energies, the CTMC method provided a good description of the formation and resulting symmetry of the "electron-captureto-the-continuum" peak, the magnitude and shift of the binary peak resulting from two-center effects, and the contribution to the spectrum coming from "saddle-point" electrons.

In order to provide a means of examining the electronic probability density ejected into the two-center continuum without the need for approximations which theory and experiment have shown to be inadequate, we have sought to directly solve the Schrödinger equation for protons colliding with atomic hydrogen on a numerical lattice. Hopefully in this context, the most clear picture of the physics underlying ion-atom collisions, and in particular ionization, can be obtained.

Earlier approaches based on this type of approach were developed to directly solve the time-dependent Schrödinger $(1,9,10)$ equation or the time-dependent Hartree-Fock $(11,12)$ equations. Both finite difference and finite elements methods have been used for discretizing the wavefunction and the actions of operators. Despite the high performance of modern supercomputers, these works indicated that the numerical study of even a simple three-body system, such as $\mathrm{H}^{+}+\mathrm{H}$, remained a difficult task. Due to the computational limits imposed by storing and operating on a very large multidimensional matrix representing the wavefunction, the earlier studies reduced the dimensionality of the problem by considering only zero impact parameter, imposing axial symmetry, or using a rotating frame (13) which would mimic the effect of a non-zero impact parameter.

In order to explore the behavior of the ionization process over a wide range of collision energies and impact parameters, we have recently (14) considered 

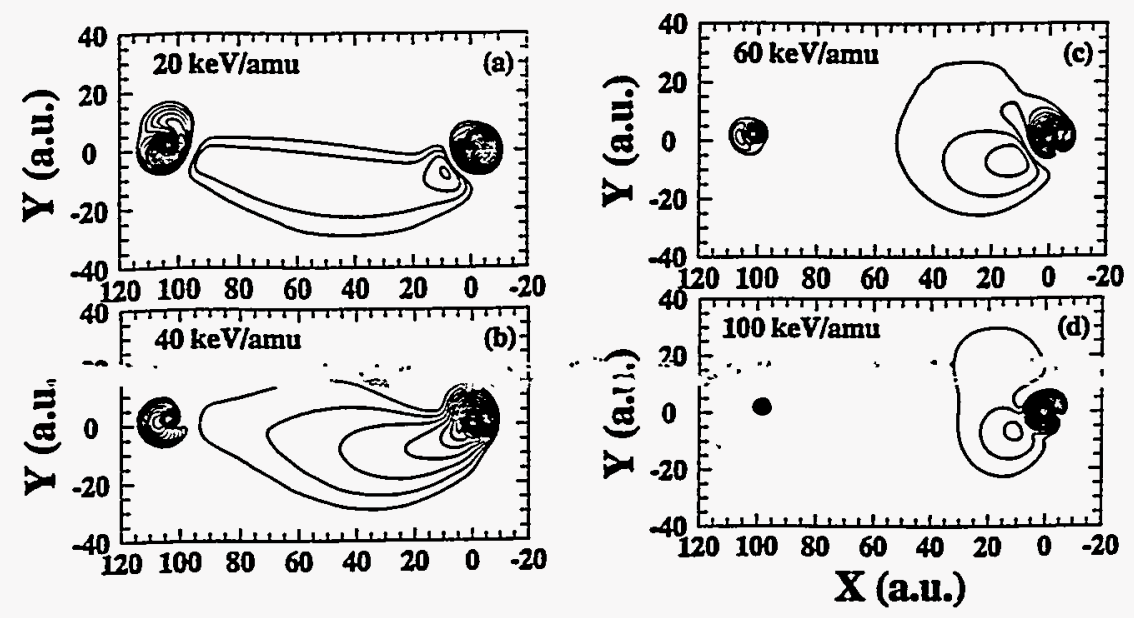

FIG. 2. The electronic probability density in 2D model collisions of a proton with atomic hydrogen displayed at a constant impact parameter (2 a.u.) for several energies long after the collision with the target.

collisions in two-dimensional (2D) Cartesian space. In addition, to ameliorate the difficulties associated with trying to represent the singular Coulomb potential on a numerical lattice of finite spacing, we introduce a model interaction based on the inclusion of a so-called softcore parameter to remove the singularity. We chose a value of the softcore parameter which yields the same binding energy as in the three-dimensional (3D) hydrogen atom, and also possesses radial and momentum distributions closely resembling those found in three dimension. Thus, the dynamics of a collision in 3D are reasonably well reproduced. By using a smaller collision volume, we have demonstrated that this is the case by performing full 3D calculations as checks. The results of simulation yield insight into the collision dynamics leading to such features as saddle point ionization, and are described in some detail below.

We solve the time-dependent Schrödinger equation in this model space using lattice techniques to obtain a discrete representation of the wavefunction, i.e. $\psi(x, y) \rightarrow \psi\left(x_{i}, y_{j}\right) \equiv \psi_{i, j}$, and all coordinate-space operators on a two- 
dimensional Cartesian mesh. Local operators such as potentials simply become diagonal matrices composed of their values at the lattice points, i.e. $V(x, y) \rightarrow V\left(x_{i}, x_{j}\right) \delta_{i, i^{\prime}} \delta_{j, j^{\prime}} \equiv V_{i, j}$. Derivative operators, such as the kinetic energy, have lattice representations in terms of matrices, i.e. $\partial / \partial x \rightarrow D_{i, j}^{(x)}$. We have implemented our solutions using both a low-order three-point finitedifference method (15), resulting in banded derivative matrices, and highorder methods such as Fourier and basis-spline collocation (16), which give full-matrix representations for the derivatives.

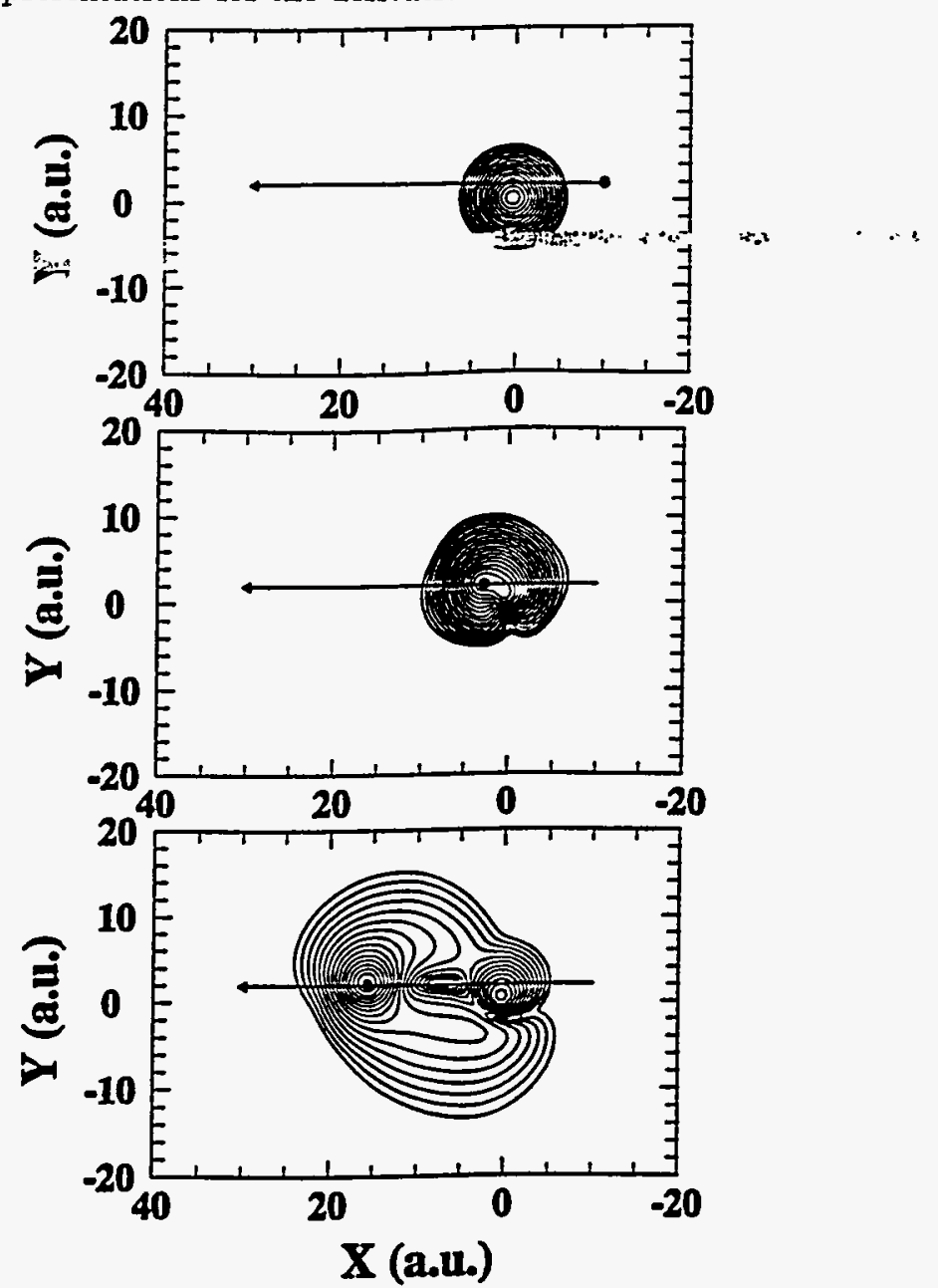

FIG. 3. The electronic probability density in a $2 \mathrm{D}$ model collision of a proton with atomic hydrogen at $10 \mathrm{keV}$ and an impact parameter of 2 a.u. (continued in Figure 4).

Figure 2 illustrates the late collision time topology of the electronic density for a fixed impact parameter (2 a.u.) for several energies $(20,40,60,100 \mathrm{keV})$ 
obtained from these simulations. Clearly seen is the transition from saddlepoint-dominated ionization at the lower energies to direct, target-centered ionization for higher energies. Also, the decrease of density on the projectile illustrates the rapid falloff of charge exchange with increasing impact energy. Also, by monitoring the overlap of the time-evolved wavefunction with a basis of target and projectile states, we have computed elastic, excitation, capture, and ionization probabilities in these collisions. Work is under way to derive the same information and visualizations in full 3D calculations.

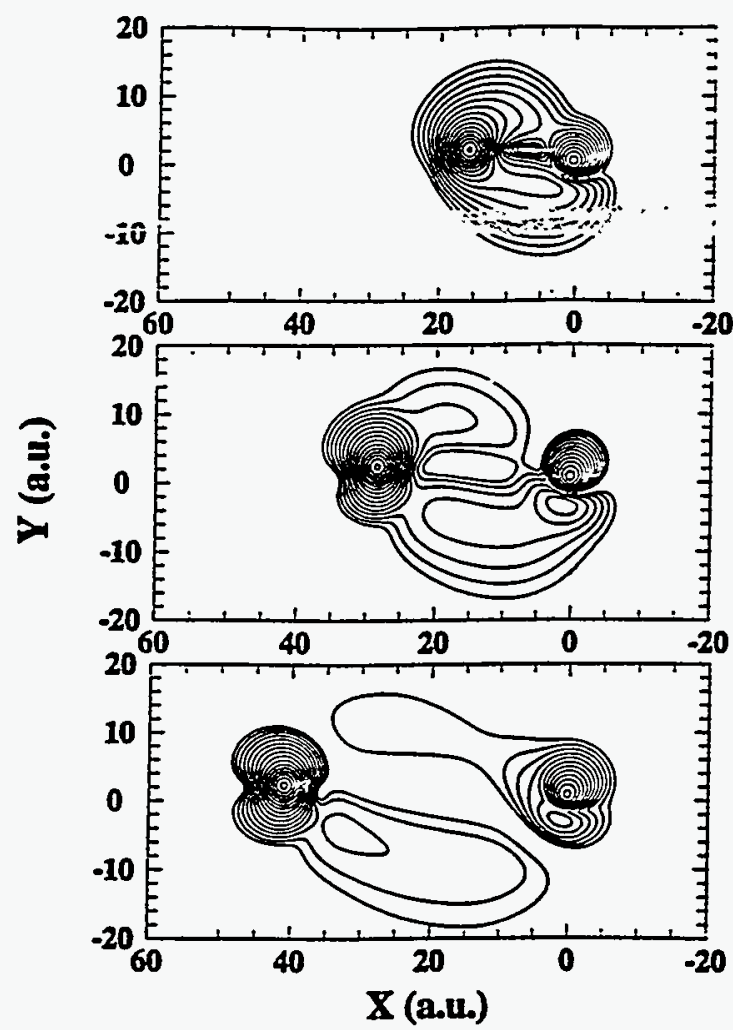

FIG. 4. The electronic probability density in a 2D model collision of a proton with atomic hydrogen at $10 \mathrm{keV}$ and an impact parameter of 2 a.u. The time slices show the evolution of a zero-density island centered about the saddle or equiforce point between the two protons. (continued from Figure 3).

Another typical result of the 2D time propagations is the case shown in Figures 3 and 4, which we describe in some detail due to the significance of the result to discussion of the production of saddle point ionization. The projectile is introduced 10 a.u. to the right of the target and moves on a straight line for 140 a.u., here with an impact energy of $10 \mathrm{keV}$ and impact parameter of 2 a.u. The first segment in Figure 3 shows the initial unperturbed state of the $2 \mathrm{D}$ hydrogen atom, while successive frames show the evolution of 
the density through an intermediate $\mathrm{H}_{2}^{+}$quasi-molecule temporarily formed in the collision to that present as the protons separate. Obvious in these frames is the large transfer of density to the projectile and what will later in Figure 4 be seen to be ejection to the continuum.

The collision is followed to larger times in Figure 4, and is fairly typical of what we find for collision energies between about 6 and $15 \mathrm{keV}$ for impact parameters in the range 0.4 to 2.6 a.u. In these frames, one sees the formation of a "zero density island" between the projectile and target in the location one would expect at first thought to see a significant density associated with the equiforce or saddle point of the projectile-target potential. This result is in qualitative agreement with a very recent description (17) for this low energy collision range, based on the so-called theory of hidden crossings.

\section{3D $\mathrm{P}^{-}+\mathrm{H}$ CuLlisions}

Motivated especially by recent experimental measurements (18) of the total cross section for ionization of atomic hydrogen by antiproton-impact, we have applied our 2D and 3D lattice approaches for proton-hydrogen scattering to this new system. A typical result of the 2D time-evolution of the electronic probability density is shown in Figure 5 for a collision energy of $10 \mathrm{keV}$. The most striking feature is the development of a large region surrounding the antiproton from which electronic density is repelled. This feature is the analog of the electron-capture-to-the-continuum cusp found in positive particle impact, and has been termed the "anti-cusp."

By computing the overlap of the time-evolved wavefunction with various target states as the collision progresses, we can determine the probability that the target will be ionized. Figure 6 shows these probabilities as a function of time for a full $3 \mathrm{D}$ collision at $1 \mathrm{keV}$ for three different impact parameters. This figure shows that the probability of ionization rises sharply at a point in time coinciding with the antiproton passing the distance of closest approach to the target nucleus. Owing to the unitarity of the channel probabilities, the elastic and excitation probability falls in complement to the rise in ionization. At long times the probabilities stabilize and may be integrated to yield total channel cross sections. This figure also shows the impact parameter (b) dependence of the channel probabilites. For example, at $b=0.3$ a.u., the ionization probability is almost ninety percent, but drops to just over fifteen percent by $b=1.8$ a.u.

By time-evolving the initial $H(1 s)$ wavefunction in the presence of the impinging antiproton for four impact energies with approximately 10 to $15 \mathrm{im}-$ pact parameters per energy, we have obtained preliminary total ionization cross sections between 1 and $500 \mathrm{keV}$. These are displayed in Figure 7 along with the experimental measurements of Knudsen et al. (18) and various other theoretical approaches. The antiproton-hydrogen system is of particular interest in that it is the simplest atomic collision, involving only three parti- 


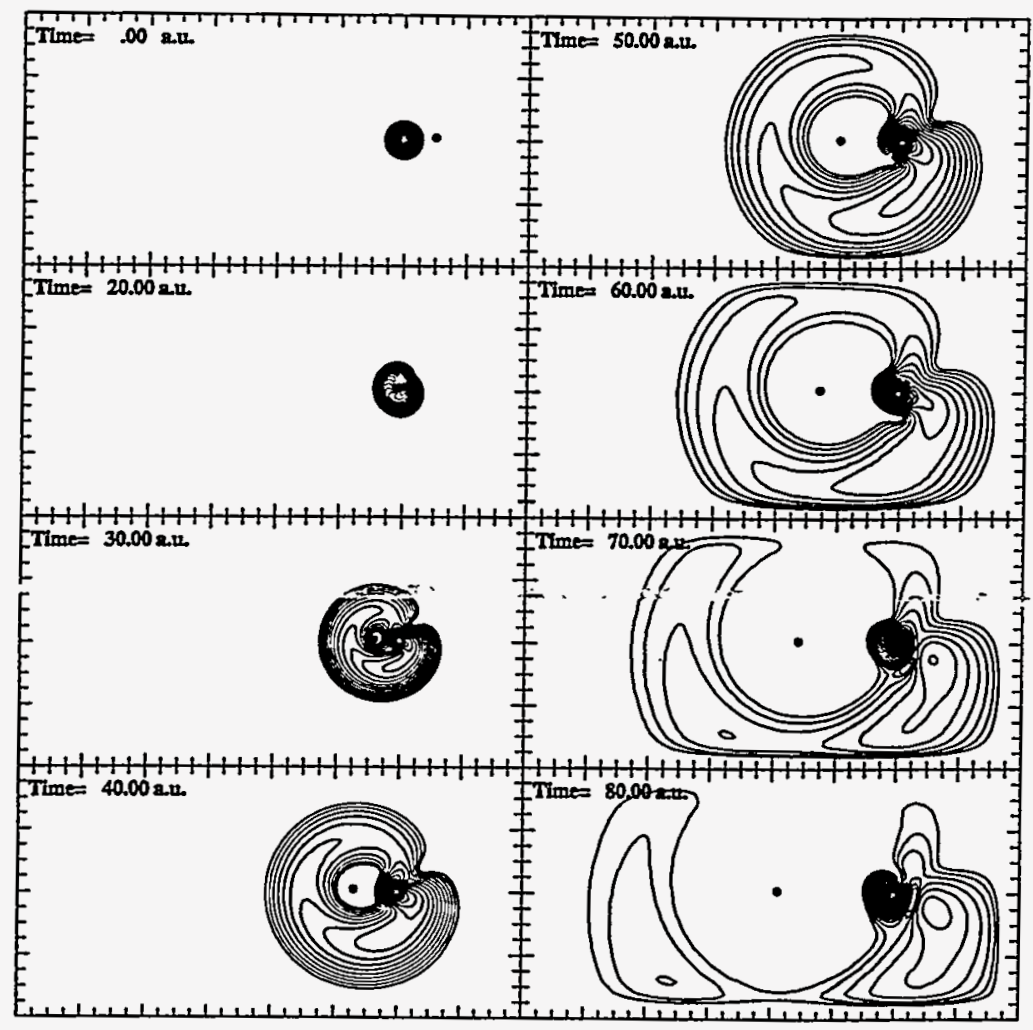

FIG. 5. The electronic probability density in 2D model collisions of an antiproton with atomic hydrogen at $10 \mathrm{keV}$ and an impact parameter of 1 a.u. The time slices show the formation of an anti-cusp centered on the projectile, the anaiog of the electron-capture-to-the-continuum peak in proton-atom collisions.

cles, and therefore provides a fundamental test of theory. In particular, it is simpler than electron- or proton-impact of atomic hydrogen since neither a two-electron wavefunction nor the charge transfer channel need be treated. The absence of these effects also lead to the result that the ionization cross section does not drop at low collision energy. In Figure 7 we show the low energy limits of the ionization cross section for this system imposed by the Fermi-Teller (FT) model (19) and our recent hidden crossings (HC) model (20). Also shown are the close coupling (CC) results of Toshima (21) and our classical trajectory Monte Carlo (CTMC) and continuum-distorted-waveeikonal-initial-state (CDW-EIS) calculations. The 3D lattice (s3D) calculations reproduce very well the behavior of the cross section over a wide range of collision energies. 


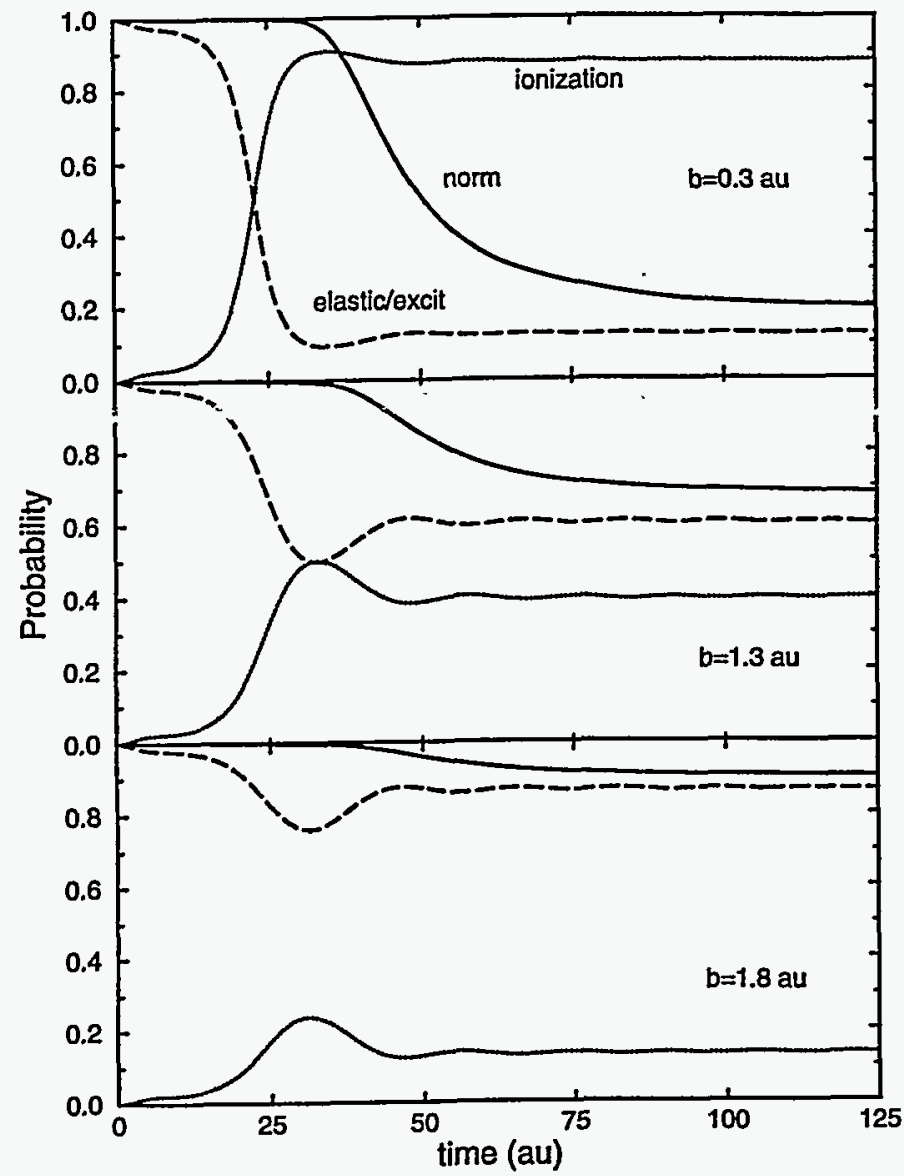

FIG. 6. The probability for various processes as a function of collision time for three impact parameters in full 3-dimensional calculations for antiprotons colliding with atomic hydrogen at $1 \mathrm{keV}$. The wavefunction is absorbed at the edges of the numerical lattice and thus the norm as depicted drops when there is a large amount of density ejected to the continuum. The dashed curve gives the sum of elastic and excitation to low-lying states and the dotted curve shows the ionization probability. 


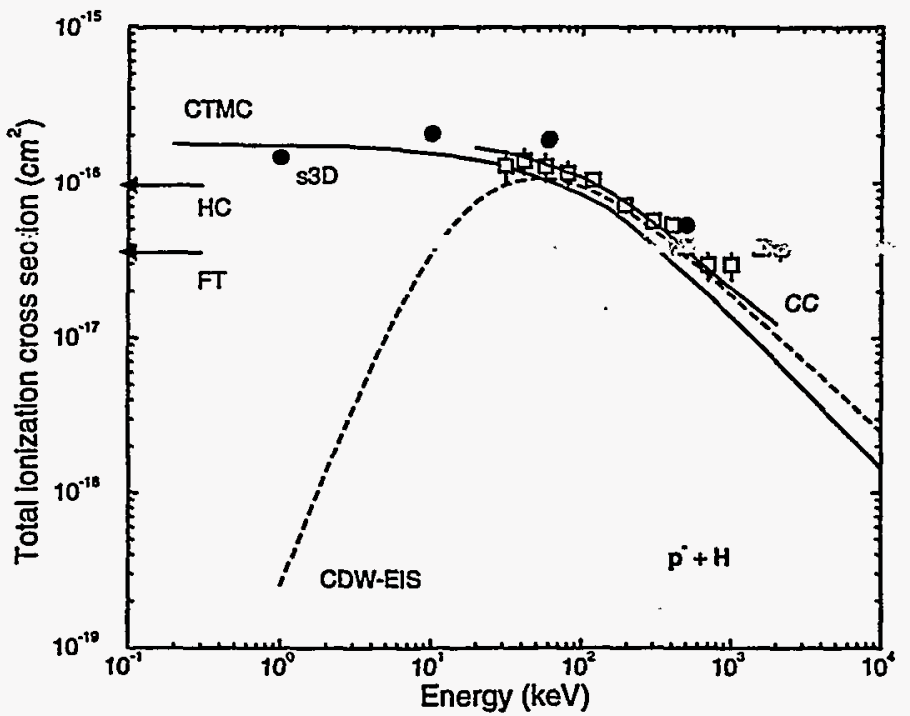

FIG. 7. The total cross section for ionization in antiproton collisions with atomic hydrogen. The experimental points are from Knudsen et al. (18). The theoretical methods denoted by CTMC, CDW-EIS, etc.are described in the text. The present 3-dimensional lattice calculations are given by the filled circles (s3D).

\section{FULLY CORRELATED TWO-ELECTRON SYSTEMS}

Another type of fundamental atomic three-body system is that composed of a heavy particle and two electrons. Rather than possessing the difficulties associated with describing an electron moving in a two-center field as in the $p^{+}, p^{-}+H$ problems discussed above, complete description of this system involves accurately treating the electron-electron interaction. A sketch of how such fully correlated two-electron systems could be treated on a numerical lattice was given by Bottcher (22) several years ago at ICPEAC XVI. Since then, we have completed the development of this technique (23), have applied it to a number of basic problems $(23,24)$, and are investigating several others. These include structure calculations of the ground, singly, and doubly excited states of $\mathrm{He}, \mathrm{H}^{-}$, and two-, or psendo-two-electron ions, the time-dependent description of autoionization, electron impact excitation and ionization of $\mathrm{H}$, 
and the photoexcitation and photoionization of He and Be. All of these systems are treated with the same underlying technique, the direct solution of the fully correlated two-electron Schrödinger equation on a numerical lattice.

Key to efficiently implementing this method is the ability to describe the two electrons in a reduced number of coordinates and through the solution of only a small number of coupled partial differential equations. In this approach, the wavefunction takes the form (23)

$$
\Psi\left(\hat{r}_{1}, \hat{r}_{2}\right)=\sum_{l=w}^{L} \psi_{l}\left(r_{1}, r_{2}, \vartheta\right) \mathcal{Y}_{l, L+w-l}^{L M}\left(\hat{r}_{1}, \hat{r}_{2}\right),
$$

where $\vec{r}_{1}$ and $\vec{r}_{2}$ are vectors locating the two electrons, $\vartheta$ is the angle between $\vec{r}_{1}$ and $\vec{r}_{2}, L$ and $M$ are the total orbital angular momentum and its projection, $\varpi$ is the parity; and $\mathcal{V}$ denntes a rounled snherical harmonic. For example. for a ${ }^{1} S$ state such as the ground state of helium, the sum contains only one term. Thus, the simplification of the problem is the reduction of the formally infinite sum over coupled spherical harmonics to a remarkably few terms, and of the full six-dimensional space (e.g. $r_{1}, \theta_{1}, \phi_{1}, r_{2}, \theta_{2}, \phi_{2}$ ) to three dimensions $\left(r_{1}, r_{2}, \vartheta\right)$.

By using the variational procedure, relatively straightforward angular momentum algebra allows the Schrödinger equation to be replaced by a set of coupled equations involving the expansion coefficients $\psi_{l}\left(r_{1}, r_{2}, \vartheta\right)$,

$$
\left(h_{1}+h_{2}+h_{\vartheta}+\frac{1}{r_{12}}-E\right) \psi_{l}+\sum_{l^{\prime}=w}^{L}\left(\mathcal{U}_{\eta^{\prime \prime l}}^{(1)}+U_{\eta^{\prime} l}^{(2)}\right) \psi_{l^{\prime}}=0
$$

where

$$
\begin{aligned}
h_{p} & =-\frac{1}{2} \frac{\partial^{2}}{\partial r_{p}^{2}}-\frac{Z}{r_{p}}+\frac{l_{p}\left(l_{p}+1\right)}{2 r_{p}^{2}}, \\
h_{\vartheta} & =\left(\frac{1}{r_{1}^{2}}+\frac{1}{r_{2}^{2}}\right)\left(-\frac{1}{2} \frac{1}{\sin \vartheta} \frac{\partial}{\partial \vartheta} \sin \vartheta \frac{\partial}{\partial \vartheta}\right) \\
u_{l^{\prime} l}^{(p)} & =-\frac{\mathcal{Z}_{l^{\prime} l}^{(p)}(\vartheta)}{\mathcal{Z}_{l^{\prime} l}^{(0)}(\vartheta)} \frac{1}{r_{p}^{2} \sin \vartheta} \frac{\partial}{\partial \vartheta}
\end{aligned}
$$

where $p=1,2, r_{12}=\left(r_{1}^{2}+r_{2}^{2}-2 r_{1} r_{2} \cos \vartheta\right)^{\frac{1}{2}}$, and $Z$ is the charge of the nucleus. The so-called $\mathcal{Z}$-coefficients, $\mathcal{Z}^{(0)}, \mathcal{Z}^{(1)}$, and $\mathcal{Z}^{(2)}$, are functions of $\vartheta$ and are given in Reference (23) as matrix elements of various angular momentum operators connecting coupled spherical harmonics. We represent the wavefunction and the action of the various operators utilizing the basis-spline collocation method.

In this case, the basis-splines, which incorporate the boundary conditions required, are used to expand the coefficients $\psi_{l}$ in a product, $u_{i}\left(r_{1}\right), u_{j}\left(r_{2}\right), w_{k}(\vartheta)$, i.e. 
TABLE 1. The table lists the computed expectation values of total energy along with accurate reference values for states of $\mathrm{He}, \mathrm{H}^{-}$, and Be. For the doubly excited state, the decay width is reported as the imaginary part of the energy. All quantities are given in atomic units.

\begin{tabular}{|l||l|l|}
\hline State & $\langle E\rangle_{\text {reference }}$ & $\langle E\rangle$ \\
\hline $\mathrm{He}\left(1 s^{2}\right)^{1} S$ & -2.90372 & -2.9032 \\
$\mathrm{He}(1 s 2 s)^{1} S$ & -2.14597 & -2.1448 \\
$\mathrm{He}(1 s 2 p)^{1} P$ & -2.12384 & -2.1234 \\
$\mathrm{He}(1 s 3 d)^{1} D$ & -2.05563 & -2.052 \\
\hline $\mathrm{He}\left(2 s^{2}\right)^{1} S$ & $-0.787-i 0.0024$ & $-0.77881-i 0.00228$ \\
\hline $\mathrm{H}^{-1} S$ & -0.52775 & -0.5265 \\
\hline $\mathrm{Be}\left(1 s^{2} 2 s^{2}\right)^{1} S$ & -1.003 & -1.011 \\
\hline
\end{tabular}

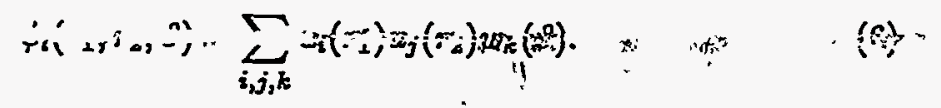

Then the principle of collocation is applied in which we require the residual to vanish at a set of points, called collocation points, intermediate to the knots. Since we use a set of knots which is not uniformly spaced in order to have a good density of points where the wavefunction has its greatest density, the resulting lattice representation of the Hamiltonian is not Hermitian. Therefore, we compute in addition to the wavefunction, $\Psi$, its adjoint, $\Phi$, so the vectors $\Psi$ and $\Phi$ are biorthogonal, and norms, expectation values, and other operators will be properly defined.

By utilizing methods of partial eigensolution $(22,23)$ (since the rank of the Hamiltonian is too large for practical diagonalization), we can begin with a trial wavefunction, such as the product of hydrogenic $1 s$ orbitals, and iteratively approach the true ground state of helium. The accuracy of this approach is ultimately limited by the number of basis-splines that we can incorporate into the expansion of the wavefunction, but with a reasonably small number (e.g. 78, fifth-order basis splines on a mesh extending from 0 to 6 a.u.), we have been able to obtain an accuracy of about 0.02 percent with about 1500 iterations of the damped relaration. A summary of our results for the ground and several low-lying excited states of He is given in Table 1. In addition, we show trial results for the ground state of $\mathrm{H}^{-}$and $\mathrm{Be}$, the latter calculation utilizing a pseudo-potential to simulate the presence of the core electrons. Finally, the table gives our result for the energy and decay width of a doubly excited state of He, determined by spectral analysis of the timedependent overlap of the initial state with the time-evolved state. These tests have helped us to validate the theoretical and numerical techniques which we have developed to treat the fully correlated two-electron problem.

Also, plots of the probability density obtained using the lattice wavefunction can give insight into the effects of correlation, for the structure of helium, or more importantly for the description of time-dependent processes. Figure 8 shows, for example, that for the ground state, the electrons are most likely to 
be found at equal radial distances, since the density is mostly peaked along the line $r_{1}=r_{2}$, while the greatest probability is for the electrons to have an angle between their position vectors near $\pi$, owing to their mutual repulsion. In addition, for small values of $\vartheta$, the density peaks on either side of the line $r_{1}=r_{2}$, indicating that at small angular separations, one or the other electron is pushed inward (outward) from the other due to the repulsion. All these simple observations are clearly in accord with our intuitive picture of the consequences of electron-electron interaction in helium.
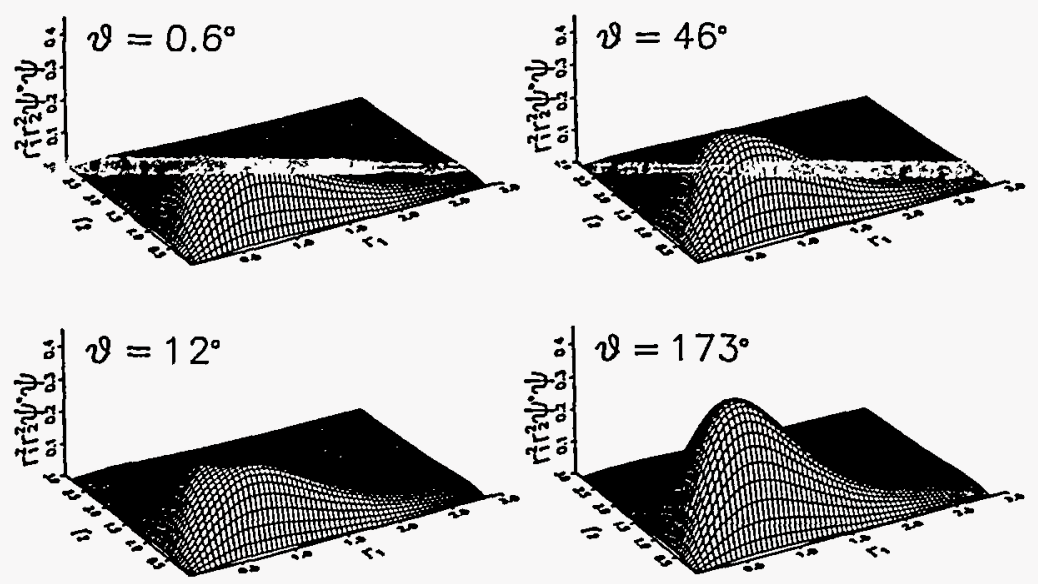

FIG. 8. The probability density in atomic units for the ground state of helium computed using the expansion coefficient $\psi_{0}\left(r_{1}, \tau_{2}, \vartheta\right)$ for several values of $\vartheta$.

We have also used the present lattice approach to follow up much earlier related models which used restricted dimensions, model wavefunctions, and less sophisticated numerical procedures (25) regarding the electron impact of atomic hydrogen. In this case, we represent the incoming electron by a wavepacket of a given angular momentum and time-propagate the total wavefunction through the collision. Figure 9 shows the electronic probability density at several time steps for a collision energy of $27.2 \mathrm{eV}$ and total angular momentum of $\mathrm{L}=0$. The left column is for the singlet configuration and the right column for the triplet. The axes are the radial coordinate of either electron (i.e. $r_{1}$ and $r_{2}$ ) and the results are plotted for an interparticle separation of $\vartheta=\pi$. One can see the development of a ridge of density in the continuum for the singlet case (along the line $r_{1}=r_{2}$ ) and the exclusion from this ridge for the triplet case. By monitoring the overlap of the time evolved wavefunction with a basis of target states, elastic, excitation, and ionization probabilities can be computed. In Table 2 we compare probabilities computed in this way with results of the second-order distored-wave Born approximation regarding elastic scattering, finding generally very good agreement. We 

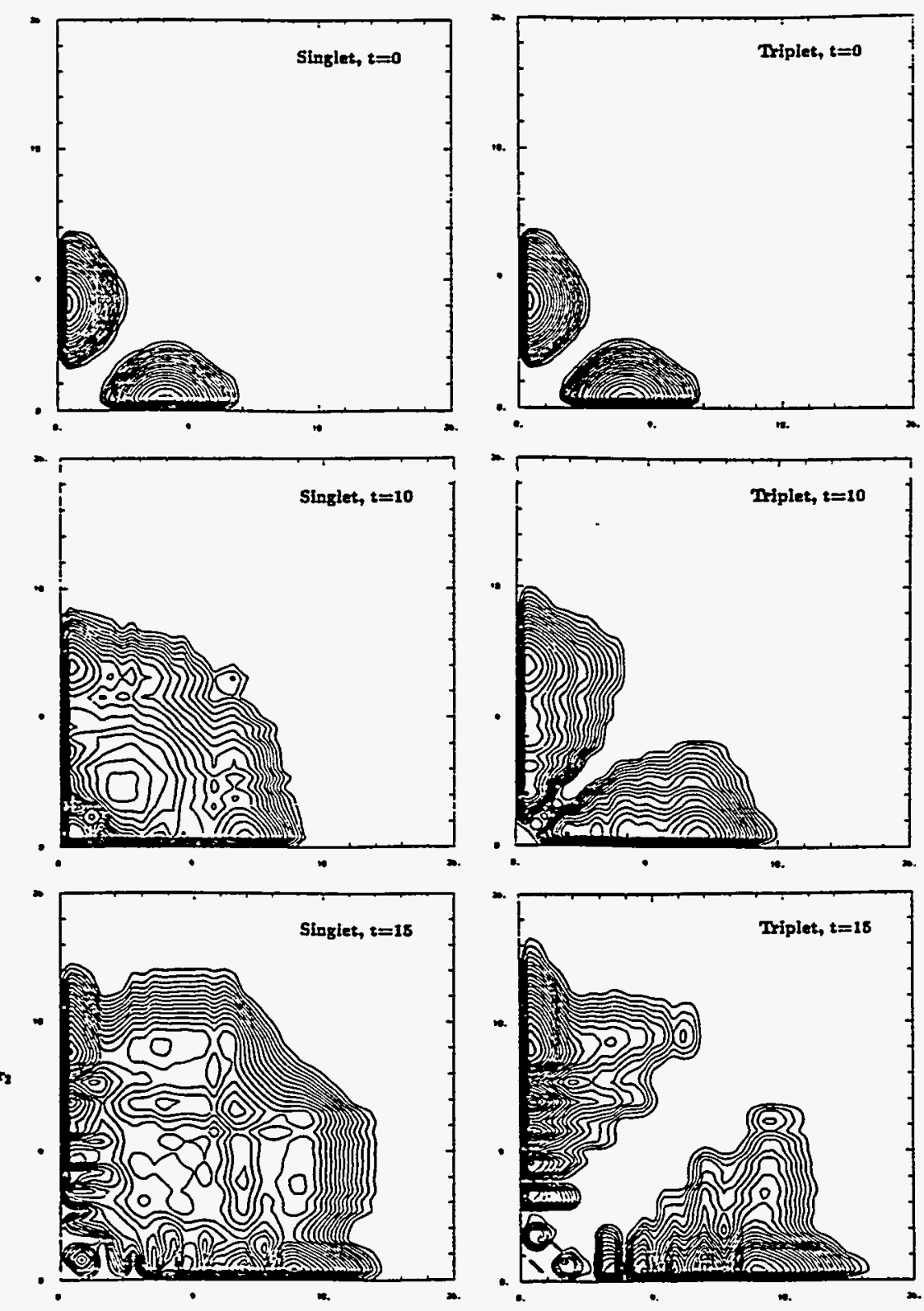

$x_{1}$

FIG. 9. The electronic probability density computed as the square of the coefficient $\psi_{l}\left(r_{1}, r_{2}, \vartheta\right)(\mathrm{Eq}$. 6) for $27.2 \mathrm{eV}$ electron-impact of atomic hydrogen. The total angular momentum is zero, $\vartheta \approx \pi$, and the two columns show the result for either singlet or triplet symmetry. Times are given in atomic units, the projectile electron being represented by a wavepacket initially centered at 7.5 a.u. from the target center. 
will follow up this preliminary calculation with computations on a finer and larger numerical grid and extend it to larger $L$ so as to obtain convergence of the total cross sections.

TABLE 2. The absolute value of the transition probability as a function of total angular momentum for $27.2 \mathrm{eV}$ electron impact of atomic hydrogen. Listed are the results for elastic scattering. Preliminary calculations using the fully correlated two-electron lattice approach are labeled "lattice" and are compared with results of the second-order distorted-wave Born approximation ("DWB2").

\begin{tabular}{|l||l|l|l|c|}
\hline \multicolumn{4}{|c|}{$\mathrm{e}+\mathrm{H}(1 \mathrm{~s}) \rightarrow \mathrm{e}+\mathrm{H}(1 \mathrm{~s})$} \\
\hline $\mathrm{L}$ & Singlet (lattice) & Singlet (DWB2) & Triplet (lattice) & Triplet (DWB2) \\
\hline 0 & 0.58 & 0.596 & 0.96 & 0.980 \\
1 & 0.12 & 0.148 & 0.41 & 0.424 \\
\hline
\end{tabular}

Work is also in progress to treat the photo-excitation and ionization of twoelectron atoms and ions as well as electron impact in a electromagnetic field. We also note that other groups have been pursuing somewhat similar lattice approaches for computing scattering phase shifts $(26,27)$, wavepacket scattering from hydrogen $(\mathrm{L}=0)(28)$, and a model atom approach (2-dimensional) for autoionization $(29,24)$, a comprehensive critique of which is beyond the scope of this progress report.

\section{CONCLUSIONS}

It has been our recent goal to develop and apply time-dependent, lattice approaches to the description of atomic collisions. These methods possess the advantage of providing a great deal of insight through the easy visualization of the collision process in time. In addition, they provide a powerful method of studying strongly interacting systems by directly solving in a nonperturbative way the relevant equations of motion (e.g. the Schrödinger or Hartree-Fock equations). Lattice techniques are now being routinely applied to study atomic collisions and are indeed becoming a part of the standard technology employed by the atomic physicist.

\section{ACKNOWLEDGEMENTS}

The research reported on here has been supported by the U.S. Department of Energy, Office of Basic Energy Sciences through Contract Number DEAC05-84OR21400 managed by Lockheed Martin Energy Systems, Inc. The author wishes to also acknowledge the collaboration of the following colleagues who have been partners in various aspects of the works reported on here: Chris Bottcher, Don Madison, Gavin Buffington, Jerry Peacher, Mitch Pindzola, Panos Gavras, Jack Wells, Carlos Reinhold and Predrag Krstic. 


\section{REFERENCES}

1. C. Bottcher, Phys. Rev. Lett. 48, 85 (1982).

2. D.S.F. Crothers and J.F. McCann, J. Phys. B 16, 3229 (1983).

3. R.E. Olson, Phys. Rev. A 33, 4397 (1986).

4. N. Stolterfoht, D. Schneider, J. Tanis, H. Alterrogt, A. Salin, P.D. Fainstein, R. Rivarola, J.P. Grandin, J.N. Scheurer, S. Andraimonje, D. Bertault, and J.F. Chemin, Europhys. Lett. 4, 899 (1987).

5. P.D. Fainstein, V.H. Ponce, and R.D. Rivarola, J. Phys. B 21, 287 (1988).

6. C.O. Reinhold and R.E. Olson, Phys. Rev. A 39, 3861 (1989).

7. R.E. Olson and A. Salop, Phys. Rev. A 16, 531 (1977).

8. J.H. Macek, J. Phys. B 24, 5121 (1991) and references therein.

9. V. Maruhn-Rezwani, N. Grün, and W. Scheid, Phys. Rev. Lett. 43, 512 (1979).

10. N. Grün, A. Mühlhans, and W. Scheid, J. Phys. B 154043 (1982).

11. K.C. Kulander. D.K.R. Sandhra, and S.E. Koonin, Phys. Rev. A 25, 2968 (1982).

12. J.D. Garcia, Nucl. Instr. and Methods in Phys. Res. A 240, 552 (1985).

13. N.H. Kwong, K.J. Schaudt, and J.D. Garcia, Comput. Phys. Commun. 63, 171 (1991).

14. P. Gavras, M.S. Pindzola, D.R. Schultz, and J.C. Wells, Phys. Rev. A (1995), to be published.

15. M.S. Pindzola and C. Bottcher, Laser Phys. 3, 748 (1993); M.W. Pindzola, T.W. Gorczyca, and C. Bottcher, Phys. Rev. A 47, 4982 (1993).

16. J.C. Wells, V.E. Oberacker, M.R. Strayer, A.S. Umar, Int. J. Mod. Phys. C 6, 143 (1995); A.S. Umar, J,-S. Wu, M.R. Strayer, and C. Bottcher, J. Comp. Phys. 93, 426 (1991).

17. S.Yu. Ovchinnikov and J.H. Macek, Phys. Rev. Lett. (1995) to be published.

18. H. Knudsen, U. Mikkelsen, K. Paludan, K. Kirsebom, S.P. Moller, E. Uggerhoj, J. Slevin, M. Charlton, and E. Morenzoni, Phys. Rev. Lett. 74, 4627 (1995).

19. E. Fermi and E. Teller, Phys. Rev. 72, 399 (1947).

20. P.S. Krstic, D.R. Schultz, C.O. Reinhold, and J.C. Wells (1995).

21. N. Toshima, Phys. Lett. A 175, 133 (1993).

22. C. Bottcher and M.R. Strajer, in Proceedings of the XVI International Conference on the Physics of Electronic and Atomic Collisions, edited by A. Dalgarno, R.S. Freud, P.M. Koch, M.S. Lubell, and T.B. Lucatorto, AIP Conf. Proc. No. 205 (AIP, New York, 1989), p. 658.

23. C. Bottcher, D.R. Schultz, and D.H. Madison, Phys. Rev. A 49, 1714 (1994).

24. D.R. Schultz, C. Bottcher, D.H. Madison, J.L. Peacher, G. Buffington, M.S. Pindzola, T.W. Gorczyca, P. Garras, and D.C. Griffin, Phys. Rev. A 50, 1348 (1994).

25. C. Bottcher, J. Phys. B 14, L349 (1981); C. Bottcher, J. Phys. B 15, L463 (1982); C. Bottcher, Adv. At. Mol. Phys. 20, 241 (1985).

26. J. Botero and J. Shertzer, Phys. Rev. A 46, R1155 (1992); J. Shertzer and J. Botero, Phys. Rev. A 49, 3673 (1994).

27. Y.D. Wang and J. Callaway, Phys. Rev. A 48, 2058 (1993); Y.D. Wang and J. Callaway, Phys. Rev. A 50, 2327 (1994).

28. L. Zhang, J. Feagin, V. Engel, and A. Nakano, Phys. Rev. A 49, 3457 (1994).

29. S.I Haan, R. Grobe, and J.H. Eberly, Phys. Rev. A 50, 378 (1994). 